\title{
High expression of APAF-1 elevates erythroid apoptosis in iron overload myelodysplastic syndrome
}

\author{
Shucheng Gu • Youshan Zhao • Juan Guo • Feng Xu • \\ Chengming Fei $\cdot$ Xi Zhang $\cdot$ Chao Xiao • \\ Chunkang Chang $\cdot$ Xiao Li
}

Received: 16 July 2013 / Accepted: 7 October 2013 /Published online: 19 October 2013

(C) The Author(s) 2013. This article is published with open access at Springerlink.com

\begin{abstract}
Apoptotic protease-activating factor 1 (APAF-1) is a central component of the intrinsic pathway of apoptosis. Our study aims at searching the role of APAF-1 in iron overload myelodysplastic syndrome (MDS). Erythroid apoptosis rate, mRNA expression levels of APAF-1, and caspase-9 activity were determined by flow cytometry, quantitative real-time $\mathrm{PCR}$, and colorimetric assay in MDS patients, respectively. In addition, K562 and MDS-L cell lines were incubated with different concentrations of ferric ammonium citrate (FAC) or ferric ammonium citrate + desferrioxamine $(\mathrm{FAC}+\mathrm{DFO})$ in vitro to observe the alteration in erythrocyte apoptosis rate, APAF-1 mRNA, and protein expression levels. Moreover, as control, erythroid apoptosis rate and APAF-1 mRNA expression were detected after silencing APAF-1 expression by endoribonuclease-prepared small interfering RNAs (esiRNAs) in K562 and MDS-L cell lines. Both erythroid apoptosis rate and APAF-1 mRNA expression of the iron overload (IO) group were significantly higher than those of the non-IO group $(P<0.001$ and $P<0.001)$. There is a significant difference of caspase- 9 activity between the IO group and the non-IO group $(P<0.001)$. Erythroid apoptosis rate and APAF-1 mRNA expression of K562 and MDS-L cell lines significantly elevated after FAC incubation in different concentrations $(P<0.001$ and $P<0.001$ for K562; $P<0.001$ and $P<0.001$ for MDS-L), while erythroid apoptosis rate and APAF-1 mRNA expression in the FAC + DFO group declined $(P<0.001$ and $P<0.001$ for K562; $P<0.001$ and $P<0.001$ for MDS-L). After silencing of APAF-1 expression with specific esiRNAs, erythroid apoptosis rate and
\end{abstract}

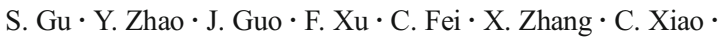

C. Chang $(\bowtie) \cdot X$. Li

Department of Hematology, Shanghai Sixth People's Hospital

Affiliated to Shanghai Jiaotong University, No.600, Yi Shan Road,

Shanghai 200233, China

e-mail: changchunkang7010@yahoo.cn
}

APAF-1 mRNA expression of K562 and MDS-L cell lines markedly decreased $(P<0.001$ and $P<0.001$ for K562; $P<0.001$ and $P<0.001$ for MDS-L). APAF-1 plays an important role in iron-induced erythroid apoptosis increase in MDS.

Keywords Myelodysplastic syndrome · Iron overload · APAF-1 $\cdot$ Erythroid apoptosis $\cdot$ esiRNAs

\section{Introduction}

Myelodysplastic syndromes (MDSs) comprise a heterogeneous group of hematopoietic stem cell malignancies characterized by peripheral cytopenias and have a substantial risk of progression to acute myeloid leukemia (AML) [1]. The most common form of cytopenia in MDS is anemia [2]. It is believed that the main cause of iron overload in MDS is repeated blood transfusion [3] and most of the patients who present with anemia become transfusion dependent during the clinical course of the disease [4].

In the human body, circulating iron is bound to transferrin. When the binding capacity of transferrin is exceeded, nontransferrin-bound iron (NTBI) species appear in the plasma [5]. NTBI contributes to the generation of reactive oxygen species (ROS). ROS-induced damage may enhance the apoptotic activity of MDS-derived hematopoietic precursors and induce mitochondrial defects and cytochrome $\mathrm{C}$ release, thus triggering apoptosis [6]. The mitochondria-dependent apoptotic pathway is involved in the mechanism of erythroid apoptosis in MDS. The apoptosome, which includes the protein apoptotic protease-activating factor 1 (APAF-1), cytochrome c/dATP, and procaspase-9, plays a central role in the apoptotic process. Triggering the death receptors or direct damage to mitochondria results in cytochrome $\mathrm{C}$ release and caspase- 9 downstream activation via APAF-1-mediated apoptosome 
formation $[7,8]$. Cytochrome $\mathrm{C}$ is released from mitochondria into the cytosol after the induction of apoptosis by many different stimuli. The released cytochrome $\mathrm{C}$ associates with APAF-1 in the presence of dATP or ATP and induces the oligomerization of APAF-1. This recognizes inactive procaspase- 9 and forms the apoptosome, triggering autocatalytic processing of procaspase- 9 . Activated caspase- 9 then processes effector caspases (caspase-3, caspase-6, and caspase-7), which in turn cause cell collapse by cleaving a specific set of substrates.

In order to study the role of APAF-1 in iron overload MDS, APAF-1 mRNA expression and erythroid apoptosis were detected in MDS patients and an iron overload model was established by using ferric ammonium citrate (FAC) in K562 and MDS-L cell lines. APAF-1 mRNA and protein expression and erythroid apoptosis rate were determined before and after FAC incubation. Moreover, deferoxamine (DFO), as control, was used to reduce the load of iron to observe the change of APAF-1 mRNA expression and erythroid apoptosis rate under iron chelation therapy. Finally, we used K562 and MDS-L cell lines with stable silencing of APAF-1 expression to further our findings in vitro.

\section{Materials and methods}

\section{Patients}

Patients diagnosed with MDS between 2010 and 2012 at the Department of Hematology in the Sixth People's Hospital affiliated to Shanghai Jiaotong University (Shanghai, China) were enrolled in this study. Thirty-five MDS patients (de novo or only treated with transfusion) were included. Written informed consent in accordance with the Declaration of Helsinki was obtained from the patients or the next of kin, caretakers, or guardians on behalf of the children participants prior to the study. Table 1 shows patients' main characteristics. Patients were categorized according to the 2008 version of the World Health Organization (WHO) Classification of MDS [9]. Two grouping strategies for prognostic stratification were adopted and carried out separately. The first strategy was based on the International Prognostic Scoring System (IPSS) [10], which defined low risk and intermediate-1 as IPSS lower-risk group and intermediate- 2 and high risk as IPSS higher-risk group. The second strategy followed the Revised IPSS (IPSS-R) [11], which defined very low risk and low risk as IPSS-R lower-risk group and the other three risk groups (intermediate-1, intermediate-2, and high risk) as IPSS-R higher-risk group. The iron overload (IO) group involved 18 patients with adjusted serum ferritin (ASF) more than $1,000 \mathrm{ng} / \mathrm{mL}$, while the non-iron overload (non-IO) group involved the remaining
17 patients [3]. APAF-1 mRNA expression in seven healthy volunteers served as a calibrator for MDS patients. The study was approved by the Ethics Committee of the Sixth People's Hospital affiliated to Shanghai Jiaotong University.

Cell culture and in vitro iron overload model

The K562 cell line obtained from American Type Culture Collection (ATCC) (Rockville, MD, USA) was cultured in RPMI-1640 medium supplemented with fetal bovine serum (FBS) and penicillin-streptomycin (PS). The MDS-L cell line gifted by Dr. Kaoru Tohyama from Kawasaki Medical School (Okayama, Japan) was cultured in RPMI-1640 medium supplemented with FBS, PS, interleukin-3 (IL-3), HEPES buffer, L-glutamine, and 2-mercaptoethanol. MDS-L was derived as a subline from a myelodysplastic cell line. MDS-L cells were positive for CD34, c-Kit, HLA-DR, CD13, and CD33, partially positive for $\mathrm{CD} 41$, and negative for $\mathrm{CD} 3, \mathrm{CD} 14, \mathrm{CD} 20$, and CD235a. The characteristics of MDS-L have previously been described in detail [13]. Although the MDS-L cell line is reported negative for $\mathrm{CD} 235 \mathrm{a}$, our preliminary experiment demonstrated a weaken expression of CD235a by flow cytometry. While MDS-L is the only cell line derived from MDS, we detected its APAF-1 expression and erythroid apoptosis assays as well.

Cells were seeded at $10^{6}$ cells $/ \mathrm{mL}$ in six-well plates and maintained at $37{ }^{\circ} \mathrm{C}$ under $5 \% \mathrm{CO}_{2}$. FAC (Sigma-Aldrich, USA) was used to set up an in vitro iron overload model. Both K562 and MDS-L cell lines were incubated with different concentrations of FAC $(0,1,2,4$, and $8 \mu \mathrm{mol} / \mathrm{L})$ for up to $24 \mathrm{~h}$. To control for the specificity of the effect of iron, $10 \mu \mathrm{mol} / \mathrm{L}$ of DFO (Novartis Pharma, Switzerland) was added to half of the wells. After incubation, the cells were washed twice with phosphate-buffered solution (PBS) for magnetic separation of $\mathrm{CD} 235 \mathrm{a}^{+}$cells and erythroid apoptosis assay. All the experiments were repeated five times.

esiRNAs mediated silencing of cell lines

Endoribonuclease-prepared small interfering RNAs (esiRNAs; Sigma-Aldrich, USA), which target APAF-1 mRNAs, were transiently transfected into K562 and MDS-L cell lines using a TransLipid Transfection Reagent (TransGen Biotech, China). Briefly, every $1 \times 10^{6}$ cells were resuspended in $1 \mathrm{~mL}$ of RPMI-1640 antibiotic-free medium and seeded in six-well plates. The esiRNA-TransLipid compound was prepared following the manufacturer's protocol. Then, the cells were incubated with $300 \mu \mathrm{L}$ of esiRNA-TransLipid compound at $37{ }^{\circ} \mathrm{C}$ under $5 \% \mathrm{CO}_{2}$. After $48 \mathrm{~h}$ of transfection, the cells were harvested. The effect of esiRNA nucleofection on specific mRNA levels was quantified by quantitative realtime polymerase chain reaction (qRT-PCR). 
Table 1 The main characteristics of MDS patients

\begin{tabular}{llll}
\hline Parameters & IO group $(n=18)$ & Non-IO group $(n=17)$ & $P$ value \\
\hline Median age, years (range) & $58(29-83)$ & $61(15-80)$ & 0.482 \\
Sex, male/female & $9 / 9$ & $10 / 7$ & 0.738 \\
Diagnosis by WHO 2008 classification (\%) & & & \\
$\quad$ RCUD/RARS/5q-syndrome/RCMD & 72 & 53 & 0.305 \\
$\quad$ RAEB-1/RAEB-2 & 28 & 47 & \\
IPSS score (\%) & & & $<0.001$ \\
$\quad$ Low/Int-1 risk & 94 & 35 & \\
$\quad$ Int-2/high risk & 6 & 65 & 0.121 \\
IPSS-R score (\%) & 7 & & $<0.001$ \\
$\quad$ Very low/low risk & 11 & 2 & $<0.001$ \\
$\quad$ Int/high/very high risk & $14.5(7-28)$ & $4(0-11)$ & $<0.001$ \\
Median months on transfusion therapy (range) & $39.5(17-86)$ & $9(0-31)$ & $<0.001$ \\
Median red blood cell units transfused (range) & $2,518.2 \pm 1,908.8$ & $452.1 \pm 224.6$ & $<0.001$ \\
ASF (mg/L) & $7.05 \pm 3.19$ & $1.95 \pm 1.23$ \\
APAF-1 mRNA expression (2 $-\Delta \Delta$ Ct) & $36.08 \pm 10.20$ & $19.32 \pm 6.39$ & \\
Erythroid apoptosis rate (\%) & &
\end{tabular}

Serum ferritin and C-reactive protein assays

Serum ferritin (SF) was detected to assess iron overload of MDS patients using standard commercial assays at the clinical laboratory of Shanghai Sixth People's Hospital. Considering that inflammation may affect the result of SF, C-reactive protein (CRP) was measured along with SF. They are both acute-phase reactants. CRP served as adjustment for SF. ASF was adopted when CRP was higher than $10.0 \mathrm{mg} / \mathrm{L}$. ASF is defined as $\mathrm{SF}(\mathrm{mg} / \mathrm{L})$ divided by the $\log 10$ of CRP $(\mathrm{mg} / \mathrm{L})$. If CRP was equal to or less than $10.0 \mathrm{mg} / \mathrm{L}$, the ASF was simply the SF [12].

\section{Magnetic separation of $\mathrm{CD} 235 \mathrm{a}^{+}$cells}

Bone marrow mononuclear cells (BMMNCs) were isolated from $7 \mathrm{~mL}$ of bone marrow samples with heparin sodium as the anticoagulant: $5 \mathrm{~mL}$ for positive selection of CD235a cells and $2 \mathrm{~mL}$ for flow cytometry assays. CD235a ${ }^{+}$mature erythrocytes and erythroid precursor cells were separated from BMMNCs with magnetic microbeads (Miltenyi Biotec, Germany), according to the manufacturer's protocol.

Flow cytometry for erythroid apoptosis assay

For erythroid apoptosis assay, a cell solution at $10^{6} / \mathrm{mL}$ was prepared by adding PBS. A total $100 \mu \mathrm{L}$ of the cell suspension was incubated with PE-conjugated anti-CD235a monoclonal antibody at room temperature away from light for $15 \mathrm{~min}$. After incubation, the cells were washed once with PBS. Then, the cells were incubated with $5 \mu \mathrm{L}$ of FITC-conjugated annexin $\mathrm{V}$ for $10 \mathrm{~min}$. Additional staining with $5 \mu \mathrm{L}$ of 7AAD was performed to discriminate dead and live cells.
The cells were analyzed by flow cytometry within half an hour on a FACS Calibur (Becton-Dickinson, USA). All antibodies were purchased from Invitrogen (Invitrogen, USA).

\section{qRT-PCR assay}

The RNA extracted from CD235a ${ }^{+}$cells using RNeazy Mini Kit (QIAGEN, Germany) served as the template for all reverse transcriptase reactions. Synthesized cDNA was obtained from $10 \mu \mathrm{L}$ of RNA via random priming, with the aid of RevertAidTM First Strand cDNA Synthesis Kit (Fermentas, Canada) following the manufacturer's protocol. The PCR totals $20 \mu \mathrm{L}$ in volume and includes the following: $2 \mu \mathrm{L}$ of cDNA solution mixed with $10 \mu \mathrm{L}$ of $\mathrm{SYBR}^{\circledR}$ Premix Ex $\mathrm{Taq}^{\mathrm{TM}} \mathrm{II}$ (TaKaRa, Japan), $0.4 \mu \mathrm{L}$ for each of forward primers and reverse primers, and $7.2 \mu \mathrm{L}$ of RNase-free water. A fluorescent quantitation PCR cycler (LightCycler, Roche, Switzerland) was used for amplification of APAF-1 and GAPDH with the following primer pairs: APAF-1 forward (for), GTCTGCTGATGGTGCAAGGA; APAF-1 reverse (rev), GATGGCCCGTGTGGATTTC; GDF15 forward (for), GTGTTGCTGGTGCTCTCGTG; GDF15 reverse (rev), CGGTGTTCGAATCTTCCCAG; GAPDH forward (for), GCACCGTCAAGGCTGAGAAC; GAPDH reverse (rev), GTGGTGAAGACGCCAGTGGA. The amplification procedure consisted of three steps: (1) denaturation at $95^{\circ} \mathrm{C}$ for $5 \mathrm{~s}$, (2) annealing at $62{ }^{\circ} \mathrm{C}$ for $20 \mathrm{~s}$, and (3) extension at $72{ }^{\circ} \mathrm{C}$ for $10 \mathrm{~s}$ (43 cycles). The threshold cycle (Ct) was subsequently determined. Expression levels of APAF-1 and GDF15 normalized to GAPDH and relative to a calibrator was expressed as $2^{-\Delta \Delta \mathrm{Ct}}$ (fold difference). Here, GDF15 was measured for predicting ineffective or apoptotic erythropoiesis. Details will be described in the part of "Discussion". 
Western blot assay

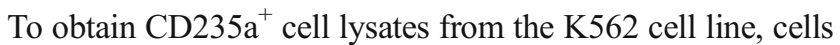
were lysed on ice for $20 \mathrm{~min}$ in a mammalian protein extraction reagent (CWBIO, China). Cell lysates were then centrifuged, and supernatants were collected. Protein concentration was measured using the BCA Protein Assay Kit (CWBIO, China). The Western blot procedure was as follows: (1) $16 \mu \mathrm{L}$ of each protein sample was loaded onto a SDS gel for electrophoresis; (2) the proteins were transferred onto nitrocellulose membranes; (3) the proteins were blocked in $5 \%$ milk and labeled with antibodies against APAF-1 (Abcam, USA), GAPDH (HuaAn Biotech, China), and relevant secondary antibodies (Abcam, USA); and (4) the proteins were visualized by enhanced chemiluminescence (Bio-Rad, USA). The films were scanned using the Epson Perfection 4490 Scanner (EPSON Europe B.V., Sweden).

\section{Colorimetric assay}

Caspase-9 is in the process of cell apoptosis signal transduction pathway. Combined with the cytochrome C released from mitochondria and APAF-1, caspase-9 can form a compound which is so-called apoptosome and activate at the same time. Activation of the key enzymes of caspase-9 can activate caspase-3, thereby promoting apoptosis signal. We used a colorimetric assay kit (Bio-Box, China) to determine caspase-9 activity of MDS patients according to the manufacturer's protocol. One unit is the amount of enzyme that will cleave $1.0 \mathrm{nmol}$ of the colorimetric substrate Ac-LEHD-pNA per hour at $37^{\circ} \mathrm{C}$ under saturated substrate concentrations.

\section{Statistical analysis}

The statistical analysis was performed using the SPSS 17.0 software (SPSS Inc., Chicago, IL, USA). Quantitative data were expressed as means $\pm \mathrm{SD}$ and tested using Student's $t$ test or analysis of variance (ANOVA). A paired sample $t$ test was used for the analysis between FAC and FAC+DFO, and FAC and FAC+esiRNAs. Pearson's correlation analysis was performed between GDF15 and APAF-1 mRNA expression. $P<0.05$ was considered as statistical significant.

\section{Results}

APAF-1 mRNA expression, caspase-9 activity, and erythroid apoptosis rate in MDS

SF still remains an essential monitoring parameter for iron overload. However, confounding factors such as iron storage, inflammation, tumor, and transfusion have to be taken into

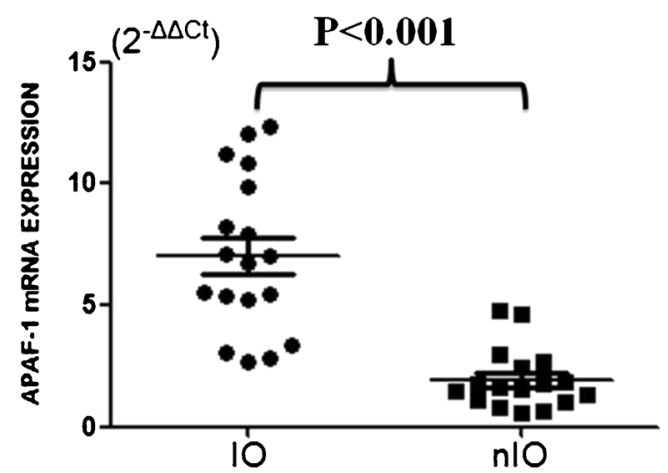

Fig. 1 APAF-1 mRNA expression of CD235a ${ }^{+}$erythroid cells in MDS patients. The APAF-1 mRNA expression of the iron overload (IO) group is $7.05 \pm 3.19$. The APAF- 1 mRNA expression of the non-iron overload $(n I O)$ group is $1.95 \pm 1.23$. There is a statistical significance of APAF-1 mRNA expression between the IO group and the non-IO group $(P<0.001)$

account. Therefore, we introduced ASF adjusted by CRP into our study to eliminate the influence of inflammation on SF.

Figure 1 shows that APAF-1 mRNA expression of the IO group is $7.05 \pm 3.19$ while that of the non-IO group is $1.95 \pm 1.23$. APAF-1 mRNA expression in the IO group was significantly higher than that in the non-IO group $(P<0.001)$. There was no statistical difference in APAF-1 expression between the IPSS lower-risk group and the IPSS higherrisk group in IO patients and non-IO patients, respectively $(P=0.695$ and $P=0.317)$; APAF-1 mRNA expression in the lower-risk group was higher than that in the higherrisk group $(P=0.004)$. No significant difference was found between the IPSS-R lower-risk group and the IPSS-R higher-risk group $(P=0.170)$. GDF15 mRNA expression levels had a statistical correlation with APAF-1 mRNA expression level in the non-IO group $(r=0.542, P=0.025)$, but there was no association between GDF15 and APAF-1 in the IO group $(r=0.170, P=0.499)$. Caspase-9 activity was used to further demonstrate the role of APAF-1 in MDS. As Fig. 2 shows, there is a significant difference of

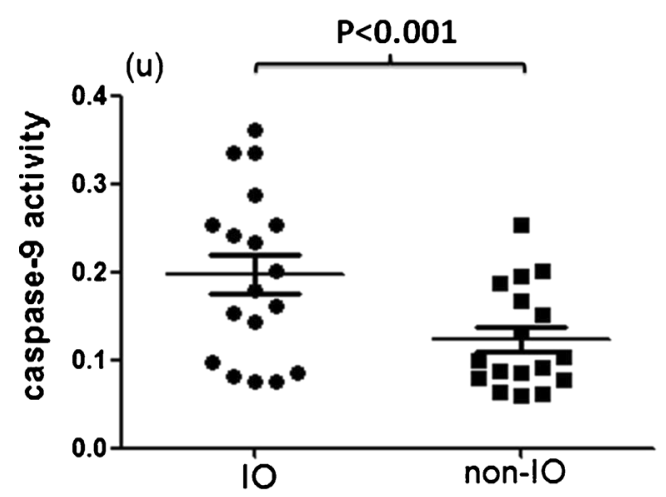

Fig. 2 The caspase- 9 activity of MDS patients. Caspase- 9 activity was used to further demonstrate the role of APAF-1 in MDS. There is a significant difference of caspase- 9 activity between the IO group and the non-IO group $(P<0.001)$ 


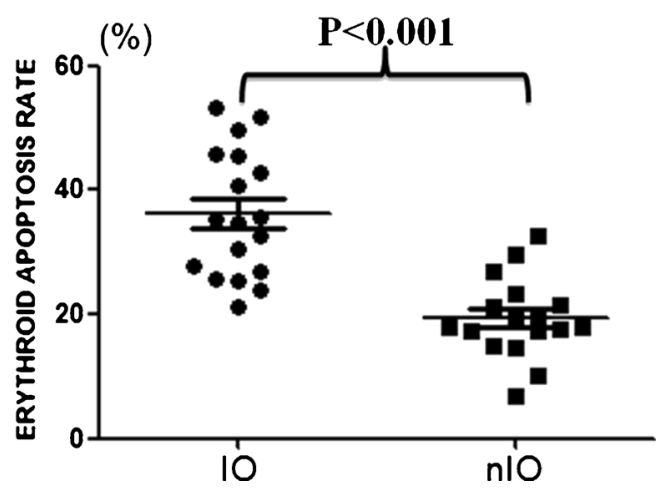

Fig. 3 The erythroid apoptosis rate of MDS patients. The erythroid apoptosis rate of the iron overload $(I O)$ group is $36.08 \pm 10.20 \%$. The erythroid apoptosis rate of the non-iron overload ( $n I O)$ group is $19.32 \pm 6.39 \%$. IO group patients have a higher erythroid apoptosis rate than non-IO group patients $(P<0.001)$

caspase-9 activity between the IO group and the non-IO group $(P<0.001)$.

The erythroid apoptosis rate of the IO group is $36.08 \pm$ $10.20 \%$, while that of the non-IO group is $19.32 \pm 6.39 \%$. The IO group had a higher erythroid apoptosis rate than the non-IO group $(P<0.001)$ (Fig. 3).

\section{Cell lines in iron overload model}

Figure 4 shows one of the results of erythroid apoptosis assay of K562 and MDS-L cell lines after incubation with different concentrations of FAC $(P<0.001$ and $P<0.001)$. The erythroid apoptosis rate increased along with increasing FAC concentration.
Figure 5 demonstrates APAF-1 protein expression of the K562 cell line after incubation with different concentrations of FAC. With the increase of FAC concentration, APAF-1 protein expression presented a rising trend.

Erythroid apoptosis rate in K562 and MDS-L cell lines decreased after FAC + DFO incubation and silencing of APAF-1

After incubation with esiRNAs, APAF-1 mRNA expression is $88.56 \pm 1.49 \%$ knockdown in the K562 cell line while $90.27 \pm 0.39 \%$ knockdown in the MDS-L cell line. Figure 5 shows the change in erythroid apoptosis rate before and after silencing of APAF-1 in K562 and MDS-L cell lines. Incubation with $\mathrm{FAC}+\mathrm{DFO}$ induced a significant decline in apoptosis rate and mRNA expression in K562 $(P<0.001$ and $P<0.001)$ and MDS-L $(P<0.001$ and $P<0.001)$ cell lines. After silencing of APAF-1 expression with specific esiRNAs, the apoptosis rate and mRNA expression dropped markedly in K562 $(P<0.001$ and $P<0.001)$ and MDS-L $(P<0.001$ and $P<0.001)$ cell lines as well (Fig. 6).

\section{Discussion}

Many MDS patients become dependent on blood transfusions and develop transfusional iron overload, which is exacerbated by increased absorption of dietary iron in response to ineffective erythropoiesis [3]. The erythroid apoptosis of MDS, particularly sideroblastic anemia, is mediated by mitochondrial

\section{K562}

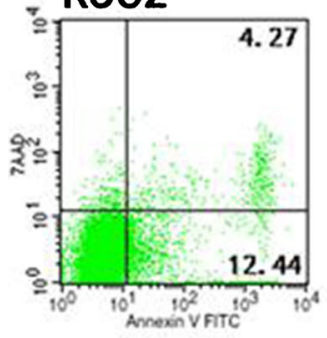

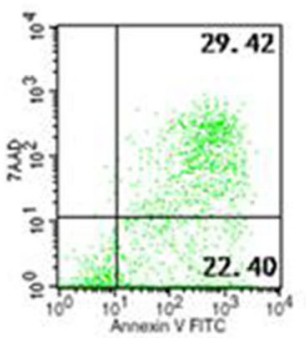
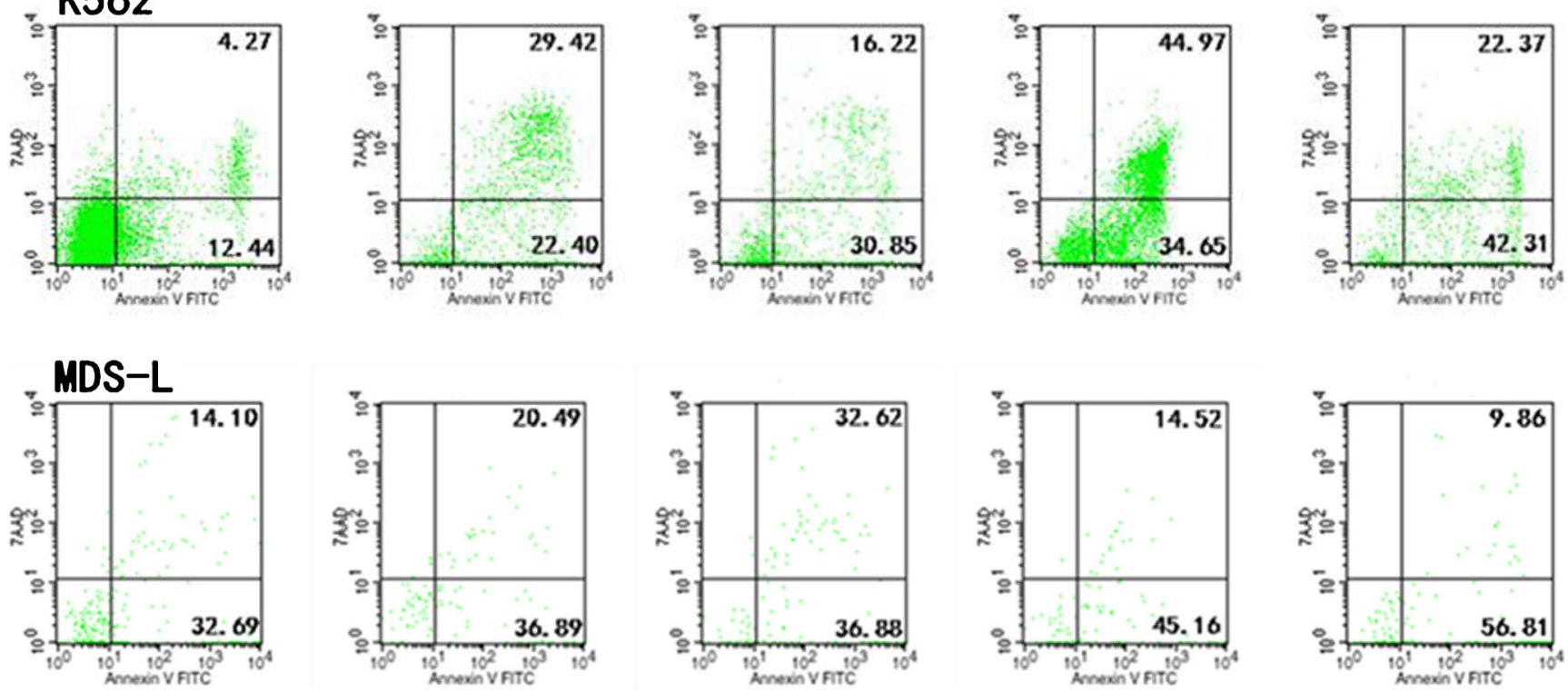

Fig. 4 The erythroid apoptosis rate of K562 and MDS-L cell lines after incubation of FAC in different concentrations. The figure shows one of the results of erythroid apoptosis assay of K562 and MDS-L cell lines after incubation of FAC in different concentrations (from left to right:

blank, $1 \mu \mathrm{mol} / \mathrm{L}, 2 \mu \mathrm{mol} / \mathrm{L}, 4 \mu \mathrm{mol} / \mathrm{L}, 8 \mu \mathrm{mol} / \mathrm{L})$. Erythroid apoptosis rate was elevating along with the increase of FAC concentration. The $P$ value is 0.001 by ANOVA 


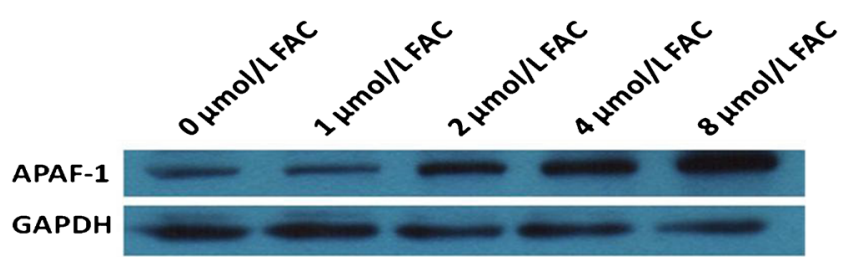

Fig. 5 The APAF-1 protein expression of the K562 cell line after incubation of FAC in different concentrations. The figure shows APAF1 protein expression of the K562 cell line after different incubation of FAC in different concentrations (from left to right: blank, $1 \mu \mathrm{mol} / \mathrm{L}$, $2 \mu \mathrm{mol} / \mathrm{L}, 4 \mu \mathrm{mol} / \mathrm{L}, 8 \mu \mathrm{mol} / \mathrm{L})$

release of cytochrome $\mathrm{C}$ which is combined with APAF-1, triggering apoptosome activation [14]. It has been reported that APAF-1 deficiency constitutes a significant mode of resistance to cytochrome C-dependent apoptosis in human leukemia cells [15]. Indeed, silencing of APAF-1 protein expression by hypermethylation of the gene promoter has been reported in several human tumors, including malignant melanoma and acute leukemia [16]. Silencing of APAF-1 expression in B cell chronic lymphoblastic leukemia (B-CLL) constitutes a negative prognostic marker in the case of concomitant p53 mutations [17]. In addition, aberrant subcellular localization of the APAF-1 protein has been demonstrated in chemoresistant B lymphoma cells, and resistance to etoposide was reversed upon APAF-1 overexpression [18]. In our study, we put emphasis on the role of APAF-1 in iron overload MDS patients.

Iron overload-induced ROS generation may cause mitochondrial activation and cytochome $\mathrm{C}$ release, which gathers APAF-1, triggering caspase-dependent apoptosis. The pathological accumulation of iron aggravates oxidative stress and mitochondrial damage, accelerating erythroid apoptosis. In our study, agreed with APAF-1 expression, erythroid apoptosis rate in the $\mathrm{IO}$ group is higher than that in the non-IO group $(P<0.001)$. Meanwhile, paralleled with APAF-1 mRNA expression, caspase-9 activity in the IO group elevated much more higher than that in the non-IO group, which suggested that APAF-1 may be the key factor to erythroid apoptosis in iron overload MDS.

Excess circulating iron is usually transformed into NTBI, eventually transported to the cell cytoplasm and stored as labile plasma iron (LPI) [3]. It had been proved that cells incubated with FAC have an increased LPI level. Therefore, we used FAC to set up an in vitro iron overload model in K562 and MDS-L cell lines. It was found that incubation with
Fig. 6 The erythroid apoptosis rate after incubation with $\mathrm{FAC}$, $\mathrm{FAC}+\mathrm{DFO}$, and silencing APAF-1 in K562 and MDS-L cell lines. The asterisk means that cell lines were only treated with esiRNAs, as blank control. To further demonstrate the role of APAF-1 in iron overload MDS, K562 and MDS-L cell lines were incubated with ferric ammonium citrate $(F A C)$, ferric ammonium citrate + deferoxamine $(F A C+D F O)$, ferric ammonium citrate + endoribonuclease-prepared small interfering RNAs $(F A C+$ esiRNAs $)$ and endoribonuclease-prepared small interfering RNAs (esiRNAs). There is a significant decrease of erythroid apoptosis rate between $\mathrm{FAC}$ and $\mathrm{FAC}+\mathrm{DFO}(P<0.001)$ and $\mathrm{FAC}$ and $\mathrm{FAC}+$ esiRNAs $(P<0.001)$ in the $\mathrm{K} 562$ cell line. There is also a significant decrease of erythroid apoptosis rate between $\mathrm{FAC}$ and $\mathrm{FAC}+\mathrm{DFO}(P<0.001)$ and $\mathrm{FAC}$ and $\mathrm{FAC}+$ esiRNAs $(P<0.001)$ in the MDS-L cell line
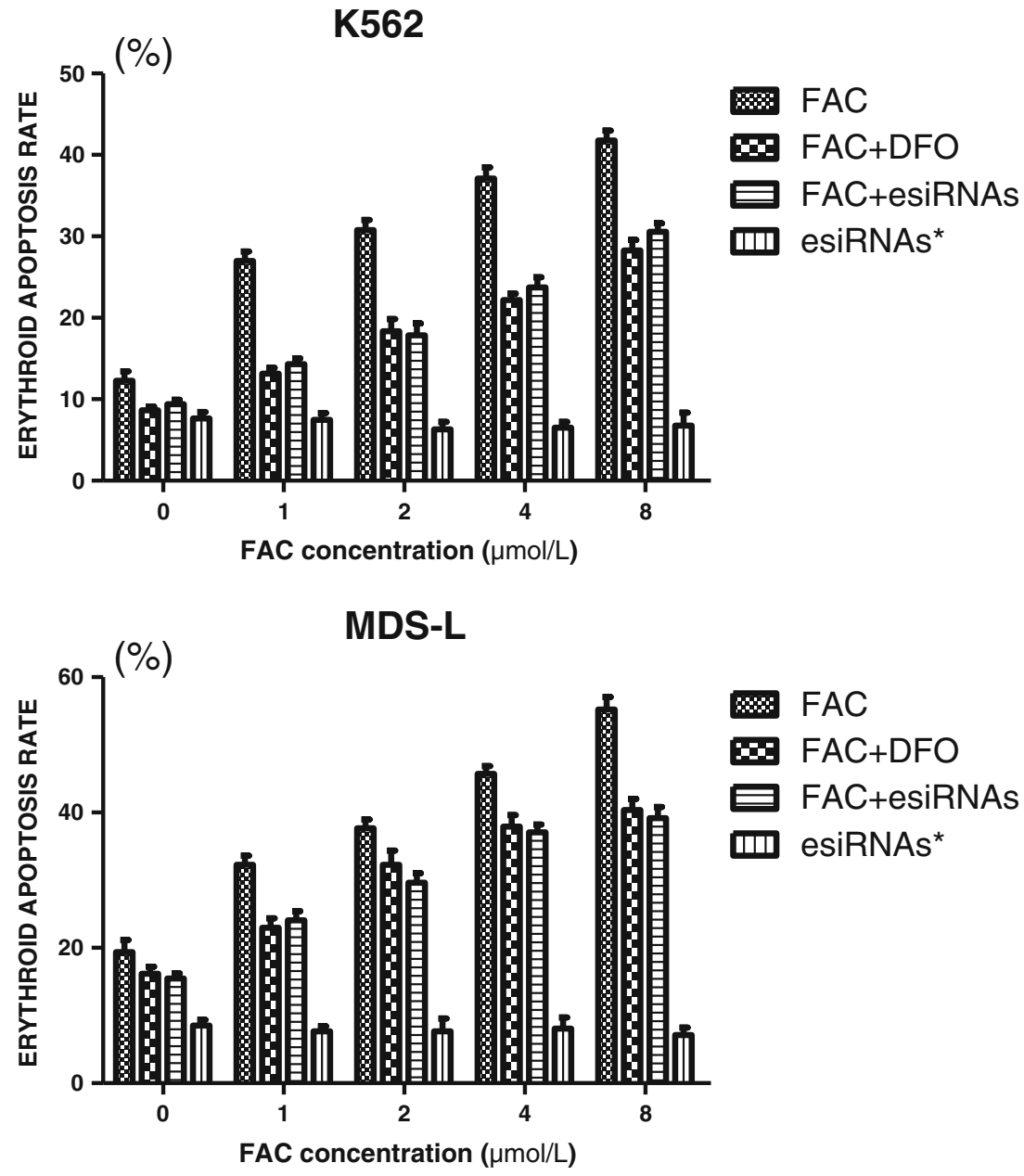
different concentrations of FAC induced erythroid apoptosis rate increase in K562 and MDS-L cell lines $(P<0.001$ and $P<0.001$ ), and what is more, the apoptosis rates increased with the increase of FAC concentrations, suggesting that increased APAF-1 expression in iron overload elevates erythroid apoptosis. In addition, Western blot analysis also demonstrated that the protein expression of APAF-1 was elevated along with increasing FAC concentration. Zermati et al. suggested that APAF-1 deficiency contributes to tumor progression not only by decreasing apoptotic caspase activation but also by reducing the DNA damage-induced cell cycle arrest, thus weakens the cytostatic effect of chemotherapy or radiotherapy [19]. It seems that ineffective erythropoiesis in iron overload may be caused by not only accelerated apoptosis but also slowed down proliferation, which might be affected by the increase of APAF-1 expression.

APAF-1 is transcriptionally and posttranslationally regulated during pathologic and physiologic conditions [20]. In order to support our findings, a group of specific esiRNAs were chosen for silencing of APAF-1 in K562 and MDS-L cell lines to guarantee the specificity of RNA interference (RNAi). After silencing of APAF-1 expression with specific esiRNAs, the apoptosis rate and mRNA expression decreased markedly in $\mathrm{K} 562(P<0.001$ and $P<0.001)$ and MDS-L $(P<0.001$ and $P<0.001)$ cell lines. This result supports that APAF-1 expression level does have a close contact with erythroid apoptosis. Furthermore, after incubation with FAC+DFO, the apoptosis rate and APAF-1 mRNA expression significantly declined in $\mathrm{K} 562(P<0.001$ and $P<0.001)$ and MDS-L $(P<0.001$ and $P<0.001)$ cell lines. When chelating the influence of iron, both APAF-1 expression and erythroid apoptosis rate reduced, indicating iron overload leading to an increase of erythroid apoptosis through APAF-1. High expression of APAF-1 elevates erythroid apoptosis in iron overload MDS.

Benites et al. demonstrated an increased APAF-1 expression during erythroid differentiation of MDS progenitor cells. Higher apoptotic rates could be a factor associated with the increased expression of APAF-1 during erythroid differentiation of CD34 $4^{+} \mathrm{MDS}$ cells [21]. Actually, some patients already had iron overload before transfusion therapy was initiated due to ineffective erythropoiesis [22]. Ineffective erythropoiesis contributes to iron overload in MDS and is correlated with an increase in intramedullary apoptosis of differentiated cells [23]. A large number of erythrocytes continue to differentiate but cannot mature until they die of apoptosis, leading to the increase of APAF-1 during erythroid differentiation progress. Tanno et al. suggested that GDF15 measurements may be helpful for predicting ineffective or apoptotic erythropoiesis [24]. We performed a Pearson's correlation analysis to review the association between GDF15 mRNA expression levels and APAF-1 expression in the IO group and the non-IO group. There was a statistical correlation of GDF15 with APAF-1 in the non-IO group $(r=0.542, P=0.025)$, but there was no association between GDF15 and APAF-1 in the IO group ( $r=0.170, P=0.499)$. In the non-IO patients, ineffective erythropoiesis might be the main cause of APAF-1 expression increase. However, the influence of ineffective erythropoiesis on APAF-1 expression in the IO group is weaker than that in the non-IO group.

In conclusion, high expression of APAF-1 in iron overload elevates erythroid apoptosis rate and may play an important role during apoptosis process in MDS.

Acknowledgments We thank Dr. Kaoru Tohyama from Kawasaki Medical School, Okayama, Japan for gifting us the MDS-L cell line. This study was supported by the National Nature Science Foundation of China (NNSFC81170463)

Open Access This article is distributed under the terms of the Creative Commons Attribution License which permits any use, distribution, and reproduction in any medium, provided the original author(s) and the source are credited.

\section{References}

1. Tefferi A, Vardiman JW. Myelodysplastic syndromes. N Engl J Med. 2009;361(19):1872-85.

2. Malcovati L, Della Porta MG, Laarakkers CM, et al. Erythroid activity, transfusion iron overload, and hepcidin levels in patient with myelodysplastic syndrome. Blood, 2008; 112: abstract 2676.

3. Gattermann N, Rachmilewitz EA. Iron overload in MDSpathophysiology, diagnosis, and complications. Ann Hematol. 2011;90(1):1-10.

4. Ambaglio I, Malcovati L, Papaemmanuil E, et al. Inappropriately low hepcidin levels in patients with myelodysplastic syndrome carrying a somatic mutation of SF3B1. Haematologica. 2013;98(3): 420-3.

5. Hershko C, Graham G, Bates GW, Rachmilewitz E. Nonspecific serum iron in thalassemia: an abnormal serum iron fraction of potential toxicity. Br J Haematol. 1978;40(2):255-63.

6. Farquhar MJ, Bowen DT. Oxidative stress and the myelodysplastic syndromes. Int J Hematol. 2003;77(4):342-50.

7. Tehranchi R, Fadeel B, Forsblom AM, et al. Granulocyte colonystimulating factor inhibits spontaneous cytochrome c release and mitochondria-dependent apoptosis of myelodysplastic syndrome hematopoietic progenitors. Blood. 2003;101(3):1080-6.

8. Hellstrom-Lindberg E, Schmidt-Mende J, Forsblom AM, Christensson B, Fadeel B, Zhivotovsky B. Apoptosis in refractory anaemia with ringed sideroblasts is initiated at the stem cell level and associated with increased activation of caspases. Br J Haematol. 2001;112(3):714-26.

9. Brunning R, Orazi A, Germing U, et al. Myelodysplastic syndromes. In: Swerdlow S, Campo E, Harris NL, et al., eds. World Health Organization classification of tumours of haematopoietic and lymphoid tissue, 4th ed. Lyon: IARC Press. 2008: 88-103.

10. Greenberg P, Cox C, LeBeau MM, Fenaux P, Morel P, Sanz G, et al. International scoring system for evaluating prognosis in myelodysplastic syndromes. Blood. 1997;89(6):2079-88.

11. Greenberg P, Tuechler H, Schanz J, et al. Revised international prognostic scoring system for myelodysplastic syndromes. Blood. 2012;120(12):2454-65.

12. Armand $\mathrm{P}, \mathrm{Kim} \mathrm{H}$, Rhodes $\mathrm{J}$, et al. Iron overload in patients with acute leukemia or MDS undergoing myeloablative stem cell transplantation. Biol Blood Marrow Transplant. 2011;17(6):852-60. 
13. Matsuoka A, Tochigi A, Kishimoto M, et al. Lenalidomide induces cell death in an MDS-derived cell line with deletion of chromosome $5 q$ by inhibition of cytokinesis. Leukemia. 2010;24(4):748-55.

14. Lindberg EH. Strategies for biology- and molecular-based treatment of myelodysplastic syndromes. Curr Drug Targets. 2005;6(6):713-25.

15. Jia L, Srinivasula S, Liu F, et al. Apaf-1 protein deficiency confers resistance to cytochrome c-dependent apoptosis in human leukemic cells. Blood. 2001;98(2):414-21.

16. Fu WN, Bertoni F, Kelsey SM, McElwaine SM, Cotter FE, et al. Role of DNA methylation in the suppression of Apaf-1 protein in human leukaemia. Oncogene. 2005;22:451-5.

17. Sturm I, Bosanquet AG, Radetzki S, Hummel M, Dörken B, et al. Silencing of APAF-1 in B-CLL results in poor prognosis in the case of concomitant p53 mutation. Int J Cancer. 2006;118:2329-36.

18. Sun Y, Orrenius S, Pervaiz S, Fadeel B. Plasma membrane sequestration of apoptotic protease-activating factor-1 in human Blymphoma cells: a novel mechanism of chemoresistance. Blood. 2005; 105:4070-7.
19. Zermati Y, Mouhamad S, Stergiou L, et al. Nonapoptotic role for Apaf-1 in the DNA damage checkpoint. Mol Cell. 2007;28(4): 624-37.

20. Fadeel B, Ottosson A, Pervaiz S. Big wheel keeps on turning: apoptosome regulation and its role in chemoresistance. Cell Death Differ. 2008;15(3):443-52.

21. Benites B, Traina F, Santos Duarte A, et al. Increased expression of APAF-1 in low-risk myelodysplastic syndrome: a possible role in the pathophysiology of myelodysplasia. Eur J Haematol. 2011;84(6): 525-30.

22. Cortelezzi A, Cattaneo C, Cristiani S, et al. Non-transferrin-bound iron in myelodysplastic syndromes: a marker of ineffective erythropoiesis? Hematol J. 2000;1(3):153-8.

23. Claessens YE, Bouscary D, Dupont JM, et al. In vitro proliferation and differentiation of erythroid progenitors from patients with myelodysplastic syndromes: evidence for Fas-dependent apoptosis. Blood. 2002;99(5):1594-601.

24. Tanno T, Noel P, Miller JL. Growth differentiation factor 15 in erythroid health and disease. Curr Opin Hematol. 2010;17(3):184-90. 